\title{
Reducing Gastroschisis Mortality: A Quality Improvement Initiative at a Ugandan Pediatric Surgery Unit
}

\author{
A. Wesonga ${ }^{1} \cdot$ M. Situma ${ }^{1} \cdot$ K. Lakhoo ${ }^{2}$
}

Published online: 21 January 2020

(C) The Author(s) 2020

\begin{abstract}
Introduction With modern treatment, survival of gastroschisis exceeds $90 \%$ in high-income countries. Survival in these countries has been largely attributed to prenatal diagnosis, delivery at tertiary facilities with timely resuscitation, timely intervention, parenteral nutrition and intensive care facilities. In sub-Saharan Africa, due to lack of these facilities, mortality rates are still alarmingly high ranging from 75 to $100 \%$. In Uganda the mortality is $98 \%$. Aim The aim of this study was to reduce gastroschisis mortality in a feasible, sustainable way using a locally derived gastroschisis care protocol at a referring hospital in Western Uganda.

Methods Data collection was performed from January to October 2018. Nursing staff were interviewed regarding the survival and management of gastroschisis babies. A locally derived protocol was created with staff input and commitment from all the team members.

Results Four mothers absconded and 17 babies were cared for using the newly designed protocol. Seven survived and were well at one month post discharge follow-up, reducing the mortality for this condition from 98 to $59 \%$. Conclusion A dedicated team with minimal resources can significantly reduce the mortality in gastroschisis by almost $40 \%$ using a locally derived protocol.
\end{abstract}

K. Lakhoo

Kokila.lakhoo@paediatrics.ox.ac.uk

A. Wesonga

anneshikanda@gmail.com

M. Situma

smssituma@gmail.com

1 Department of Surgery, Mbarara Regional Referral Hospital, Mbarara University of Science and Technology, Kabale Road, Mbarara, Uganda

2 Department of Paediatric Surgery, Childrens Hospital Level 2, University of Oxford and Oxford University Hospitals, John Radcliffe Site, Headley way, Oxford OX3 9DA, UK

\section{Introduction}

Gastroschisis is a congenital anomaly characterized by a defect in the anterior abdominal wall through which abdominal contents protrude. Gastroschisis is increasing throughout the world for unknown reasons. This condition now occurs in about 1 of every 2000-4000 live births [1].

Babies born with gastroschisis ideally should have a good prognosis and long-term survival of $>90 \%[2,3]$. With modern treatment, survival exceeds $90 \%$ in developed countries [3,4]. Survival in these countries has been largely attributed to prenatal diagnosis, delivery at a tertiary facility with timely resuscitation, timely intervention, availability of total parenteral nutrition and neonatal intensive care units and neonatal ICU. Unfortunately, these facilities and medication are always lacking in resourcelimited countries. In sub-Saharan Africa, mortality rates for 
gastroschisis still remain alarmingly high ranging from 90 to $100 \%$ [5]. In Uganda mortality for gastroschisis is $98 \%$ [6].

It could be argued that local and national government including global health funding could be better spent elsewhere, rather than on a relatively uncommon congenital anomaly like gastroschisis. However, gastroschisis is potentially curable with disability-free long-term survival achievable in the well-resourced setting [7]. Looking at disability-adjusted life years (DALYs) (DALYs = years of life lost + years lived with disability), the potentially avertable DALYs would be high, making the case for enhanced pediatric surgery capacity [8]. The same would hold for a number of other congenital anomalies, but gastroschisis is unique in terms of the rising worldwide incidence and the potential curability [9].

In the low-resource setting, there is a huge unmet burden of infectious diseases and thus a rare disease like gastroschisis is not prioritized in terms of securing parental nutrition and neonatal intensive care beds (NICU). Due to the lack of resources, we attempted to use available resources in the management of gastroschisis. The aim of this study was to reduce gastroschisis mortality in a feasible, sustainable way using a locally designed gastroschisis protocol at Mbarara hospital, a regional referral hospital in Western Uganda.

We describe an intervention to improve gastroschisis outcomes by applying a local protocol (see Table 1) using the available ward supplies and staff. The protocol was developed on the basis of the gaps identified in the study by Wesonga et al. [6], on "opportunities for improved survival for gastroschisis in Uganda" and capitalizes on aggressive resuscitation, primary bedside staged closure by a pediatric surgeon or fellow in attendance, early enteral feeds and effective team work.

\section{Aim}

To determine whether mortality of gastroschisis can be reduced by using a locally designed protocol with locally available material for the management of gastroschisis.

\section{Methods}

The medical records for gastroschisis for the past 1 year were reviewed and ward staff were interviewed regarding survival of gastroschisis for the past year.

Ward meetings were held to discuss gastroschisis mortality and how best outcomes could be improved. Knowledge, attitude and previous practices were also discussed. The published article on opportunities for improved survival in Uganda was reviewed and gaps identified [6]. The protocol was based on these gaps.

A quality improvement study team (QIST) was created from the routine ward staff that continued to perform their normal duties over and above being part of the QIST. Every team member on duty familiarized themselves with the protocol to avoid variations. Furthermore, a team member was on standby at all times in case the on call team member required assistance. The pediatric surgery fellow was the lead core team member.

\section{Intervention}

The entire ward staff and team members plus the team leader were informed as soon as a baby with gastroschisis was admitted. The role of the admitting staff member was to cover the exposed bowel with a urine bag and assist with the resuscitation of the baby. Parental involvement was actively encouraged. The protocol was instituted (Table 1).

Data were collected for demography, mortality and morbidity. Data on gastroschisis were also reviewed for a year before instituting the protocol. Staffs were interviewed regarding outcomes of gastroschisis 1 year before the start of the protocol.

\section{Ethical consideration}

Informed consent was obtained before the interventions, and continuous engagement of the parents was requested. Ethical permission was obtained from the Mbarara ethical committee.

\section{Results}

Over a 10-month period from January 2018-October 2018, a total of 21 babies were admitted to the unit with gastroschisis. Male-to-female ratio was 2.4:1. The mode of delivery was spontaneous vaginal delivery in $90 \%$ of cases. The average birth weight was $1.97(1-2.6) \mathrm{kg}$. The average time to full feeds was 14 (7-21) days. The average length of hospital stay was 17 (11-24) days. The entire bowel was reduced by the 8 th day of admission. All except two were reduced in improvised silo. One wound was covered with umbilical cord. One baby had segmental bowel necrosis, and primary closure was possible after resection and primary anastomosis. One baby had associated intestinal atresia and died on day 10 . Fourteen mothers were between 15 and 20 years old $(67 \%), 4$ were between 21 and 29 years old (19\%) and 3 were between 30 and 35 years of age $(14 \%)$.

Four mothers absconded with the babies before discharge. Seventeen babies stayed on ward until discharge or 
death. Seven babies (Table 2) were discharged from hospital, and at 1-month review they were all alive and gaining weight adequately. Ten babies died mainly at night when there is shortage of workforce (Table 3). Mortality was reduced from $98 \%$ of the previous year to $58 \%$ during year of instituting the protocol.

\section{Discussion}

The Mbarara pediatric surgical unit has a bed capacity of 17. It has two elective operating days in a week, one surgical outpatient day in a week and 24-7 emergency services. The unit sees an average of 120 outpatients in a month and does an average of 50 operations per month. The pediatric surgery workforce includes 1 pediatric surgeon, a pediatric surgery fellow and 6 nurses.

Gastroschisis has remained a challenge in Mbarara hospital with mortality of almost $100 \%$ over the past decade. Thus, it had become the norm for gastroschisis to be a non-survivable condition in this hospital. Some clinicians were still hopeful and continued to resuscitate these babies and place an improvised silo while others informed the parents of the poor of prognosis of these babies and provided palliative care.

Table 1 Protocol

Protocol

1. Insert nasogastric tube and aspirate all the stomach contents

2. If silo bag is delayed, cover the bowel with urine bag and secure with crepe bandage. Avoid placing wet swabs directly on the bowel

3. Secure IV access and give a bolus of normal saline at $20 \mathrm{mls} / \mathrm{kg}$

4. Check $\mathrm{SPO}_{2}$, if less than $90 \%$ put on $\mathrm{O}_{2}$ therapy

5 . Weigh the baby and record admission weight

6. Maintain with dextrose $10 \%$ at $4 \mathrm{mls} / \mathrm{kg} / \mathrm{h}$ (fluid overload should be avoided)

7. Intravenous ceftriaxone $50 \mathrm{mg}$ per $\mathrm{kg}$ daily for 7 days

8. Intravenous paracetamol $(10 \mathrm{mg} / \mathrm{kg})$ or rectal paracetamol $62.5 \mathrm{mg} 6 \mathrm{~h}$ for 7 days

9. Enlarge the defect generously and suture urine bag around defect at the bedside under L.A (lidocaine $1 \%$ ). Use a running suture size 3.0 or 2.0

10. Meticulous handling of gut is important during suturing the urine bag to avoid kinking of mesenteric vessels

11. Keep baby warm; place baby in the incubator and if an incubator is not available cover well with warm clothes

12. Proceed to warm saline enema about $10-20 \mathrm{ml}$ per $\mathrm{kg}$ once or twice

13. Teach mother how to aspirate nasogastric tube every $2 \mathrm{~h}$

14. Allow mother to express breast milk, soak gauze in the milk and let the baby suck the swab PRN

15 Reduce gut daily avoiding signs of abdominal compartment syndrome

16. Allow baby to breastfeed fully as soon as they start passing stool. Gradually increase as tolerated. Nasogastric tube may be clamped after several days. If the baby doesn't vomit, the tube can be remove. Supplement with IV fluids until feeding is fully established to prevent dehydration

17. Weigh the baby before discharge and record discharge weight

18. Remind mother to immunize the baby

19. Counsel the parents about the condition and possible outcomes

20. Educate the midwives to leave a long stump of umbilical cord for gastroschisis baby (this can be used to cover the defect)

Table 2 Survivors

\begin{tabular}{llllll}
\hline Patient & Weight $(\mathrm{kg})$ & Days to full feeds & Length of stay (days) & Days to closure & Outcome \\
\hline 1. & 1.2 & 16 & 21 & 4 & Home alive \\
2. & 2.8 & 12 & 16 & Primary & Home alive \\
3. & 2.2 & 18 & 21 & 7 & Home alive \\
4. & 2.5 & 8 & 24 & 6 & Home alive \\
5. & 2.8 & 16 & 18 & 5 & Home alive \\
6. & 2.4 & 13 & 15 & 8 & Home alive \\
7. & 2.0 & 11 & 15 & Home alive \\
\hline
\end{tabular}


Table 3 Non-survivors

\begin{tabular}{llll}
\hline Patient & Weight $(\mathrm{kg})$ & Length of survival (days) & Cause of death \\
\hline 1. & 1.1 & 24 & Dehydration no IVI access overnight \\
2. & 2.0 & 14 & sepsis \\
3. & 1.7 & 11 & Dehydration, no IVI access overnight \\
4. & 2.0 & 24 & High output fistula, dehydration \\
5. & 1.5 & 20 & Power cut and hypothermia overnight \\
6. & 2.1 & 11 & Sepsis \\
7. & 2.3 & 17 & Oxygen supply run out at night \\
8. & 2.0 & 23 & Oxygen supply run out at night \\
9. & 11 & No oxygen support \\
10. & 1.7 & & Power cut and hypothermia overnight
\end{tabular}

This quality improvement gastroschisis protocol translated into improved survival as evidenced by reduction in mortality from $98 \%$ pre-protocol era to $58 \%$ post-protocol era.

A Ugandan study on gastroschisis by Wesonga et al. [6] shows that the mortality rate for children with gastroschisis is $98 \%$ which is alarmingly high in the context of almost universal survival in high-income countries. We identified a number of disparities in our institution: the lack of access to prenatal ultrasound and the low accuracy of diagnosis. Without accurate prenatal diagnosis, mothers could not be counseled to deliver their babies in a hospital where pediatric surgery is available. Unfortunately, even with inhospital delivery the mortality was high. Lack of basic neonatal care, failure of early surgical management, unavailability of total parenteral nutrition and lack of central venous access are most of the contributing factors. The study concluded that many of these deaths are preventable, and improvements in both patient care and education for healthcare providers would give hope for the survival of babies with gastroschisis in Uganda.

The present quality improvement study was founded on the outcomes of this study. Much effort was put on factors that could be easily modified like resuscitation, early surgical management and early oral feeds to combat need for TPN. All improvised silos were sewn at the bedside, and thus, there was no need for operating space which was a large contributor to mortality in the previous study [6]. In the present study, we noted that most deaths occurred at night when the staffing levels were very low which is a factor to consider for the future.

In-house vs outside delivery was not an issue, since all the survivors' except one in this quality improvement study were born outside the hospital. We were able to provide adequate and early resuscitation to most of our patients noting that early resuscitation is a major predictor of mortality as described by Stevens et al. [10]. In their study, poor resuscitation was a more significant predictor of mortality rather than postnatal transfer time. The high mortality rate $(65 \%)$ was not due to lengthy transfer times but the poor clinical condition of the patients on arrival at the hospital, which relates to deficiencies in the neonatal transfer system and had a direct impact on the survival of neonates with gastroschisis.

Gastroschisis-related infant mortality in Zimbabwe is associated with well-known risk factors, such as low birth weight, prematurity and teenage mothers. However, modifiable factors identified in this study signify potential opportunities for developing innovative approaches to perinatal care in such a resource-constrained environment [11]. We have shown this successfully in our present QI study.

Multidisciplinary team training, nurse empowerment and the intentional involvement of mothers in monitoring and care provision may contribute to improving survival. The nurse-to-patient ratio Mbarara Hospital is about 1:10, and at any one point we can have 3 gastroschisis babies on ward at the same time. One of the major challenges during this QI study was inadequate nursing care. Thus, to combat this we actively involved the mothers to learn basic nursing care like nasogastric aspirations, dressings change and stopping/starting IV fluids.

The outcome of this study has had a great impact on the system and the morale of the clinical team and the community. Attitudes and practice in the management of gastroschisis has changed for the better; however, a huge challenge still remains of instituting information on the protocol across other regional referrals hospital in Uganda. The relevant health authorities are aware that gastroschisis is a survivable disease and therefore should no longer be neglected. 


\section{Limitations}

There is lack of data on mothers who absconded from hospital before discharge.

\section{Conclusion}

A locally derived gastroschisis protocol showed a reduction in mortality by almost $40 \%$. Babies born with gastroschisis can survive without TPN or ICU facilities and with minimal available resources in poor-income countries. They need a dedicated team with a positive attitude. However, additional work is needed especially in early referrals, adequate nursing staff especially on night shift and availability of stable supply of oxygen and fluid pumps. We suggest a larger multicenter study with the aim of creating a database and developing a national gastroschisis protocol.

Authors contribution All authors contributed to the study conception and design. Material preparation and data collection were performed by Ann Wesonga and Martin Situma, and analysis of data was performed by all the authors. The first draft of the manuscript was written by Ann Wisonga, and all authors commented on previous versions of the manuscript. All authors read and approved the final manuscript.

Funding This study was not funded.

\section{Compliance with ethical standards}

Conflict of interest The authors declare that they have no conflict of interest.

Informed consent Informed consent was obtained from all individual participants included in the study. Informed consent was obtained before the interventions, and continuous engagement of the parents was requested.

Ethical approval Ethical permission was obtained from the Mbarara ethical committee.

Open Access This article is licensed under a Creative Commons Attribution 4.0 International License, which permits use, sharing, adaptation, distribution and reproduction in any medium or format, as long as you give appropriate credit to the original author(s) and the source, provide a link to the Creative Commons licence, and indicate if changes were made. The images or other third party material in this article are included in the article's Creative Commons licence, unless indicated otherwise in a credit line to the material. If material is not included in the article's Creative Commons licence and your intended use is not permitted by statutory regulation or exceeds the permitted use, you will need to obtain permission directly from the copyright holder. To view a copy of this licence, visit http://creativecommons. org/licenses/by/4.0/.

\section{References}

1. Kilby MD (2006) The incidence of gastroschisis: is increasing in the UK, particularly among babies of young mothers. BMJ 332:250-251

2. DriverC BruceJ, Bianchi A et al (2000) The contemporary outcome of gastroschisis. J Pediatr Surg 35:1719-1723

3. Wright JN, Langer M, Norman ICF et al (2019) Improving outcomes for neonates with gastroschisis in low-income and middleincome countries: a systematic review protocol. BMJ Paediatr Open. https://doi.org/10.1136/bmjpo-2018-000392

4. Feldkamp ML, Botto LD, Byrne JL et al (2016) Clinical presentation and survival in a population-based cohort of infants with gastroschisis in Utah, 1997-2011. Am J Med Genet A 170:306-315

5. Ameh EA, Chirdan LB (2000) Ruptured exomphalos and gastroschisis: a retrospective analysis of morbidity and mortality in Nigerian children. Pediatr Surg Int 16:23-25

6. Wesonga AS, Fitzgerald TN, Kabuye R et al (2016) Gastroschisis in Uganda: opportunities for improved survival. J Pediatr Surg 51:1772-1777

7. BradnockTJ Marven S, Owen A et al (2011) Gastroschisis: one year outcomes from national cohort study. BMJ 343:d6749

8. Bickler S, Ozgediz D, Gosselin R et al (2010) Key concepts for estimating the burden of surgical conditions and the unmet need for surgical care. World J Surg 34:374-380. https://doi.org/10. 1007/s00268-009-0261-6

9. Bickler S, Kyambi J, Rode H (2001) Pediatric surgery in subSaharan Africa. Pediatr Surg Int 17:442-447

10. Stevens P, Muller E, Becker P (2016) Gastroschisis in a developing country: poor resuscitation is a more significant predictor of mortality than postnatal transfer time. S Afr J Surg 54:4-9

11. Apfeld JC, WrenSM Macheka N et al (2015) Infant, maternal, and geographic factors influencing gastroschisis related mortality in Zimbabwe. Surgery 158:1475-1480

Publisher's Note Springer Nature remains neutral with regard to jurisdictional claims in published maps and institutional affiliations. 